\title{
OVER THE TOP: IMPACTOS SOBRE AS REDES DOS PEQUENOS PROVEDORES DE ACESSO À INTERNET
}

\section{ARTIGO ORIGINAL}

SILVA, Antônio Eugênio ${ }^{1}$

SILVA, Antônio Eugênio. Over The Top: Impactos sobre as redes dos pequenos provedores de acesso à internet. Revista Científica Multidisciplinar Núcleo do Conhecimento. Ano 04, Ed. 09, Vol. 02, pp. 05-23. Setembro de 2019. ISSN: 24480959, Link de acesso: https://www.nucleodoconhecimento.com.br/ciencia-dacomputacao/over-the-top

\section{RESUMO}

A Internet representa uma força transformadora de paradigmas, fazendo com que situações cotidianas como a forma de assistir filmes e interagir com a sociedade tivessem uma rápida transformação nos últimos anos, impulsionadas pelas plataformas de distribuição de vídeo on-line pela Internet, que consistem em sistemas distribuídos multiplataforma formando o denominado over the top - OTT. O envio dos conteúdos de vídeo sobre a Internet necessita de diversos dispositivos de infraestrutura, associados aos Internet Service Provider - ISP, que tem de disponibilizar o acesso à Internet com largura de banda suficiente para garantir a qualidade do serviço. O crescimento do uso da Internet de banda larga gera preocupações para os ISP devido às altas demandas de banda geradas pelas aplicações OTT. A caracterização do comportamento dos usuários para serviços OTT pode contribuir para um melhor entendimento da interação dos usuários desses serviços com os ISP, o que ajudará os pequenos ISP a gerenciarem a capacidade de

1 Doutorado em Tratamento da Informação Espacial; Mestrado em Engenharia Elétrica; Especialização em MBA Empresarial - Gestão de Negócios; Especialização em Engenharia de Informações; Especialização em Engenharia em Comunicação de Dados; Especialização em Engenharia Elétrica; Graduação em Engenharia Elétrica. 
seus recursos provendo uma melhor qualidade do serviço prestado e possibilidades de oferta de novos serviços. Esse trabalho tem como objetivo avaliar, utilizando de um estudo de caso, os impactos dos serviços OTT, especificamente Netflix, Facebook e Google, em um pequeno provedor de acesso à Internet banda larga. Na pesquisa foram monitorados e coletados dados reais de todos os clientes de um ISP utilizando as ferramentas PRTG, Winbox, Wireshark e aplicativos legados nos roteadores de borda. Com o resultado do estudo de caso pode-se ter uma referencia para o dimensionamento dos enlaces de acesso ao backbone da Internet com a utilização de CDN no ambiente do ISP, pois o tráfego dos usuários no acesso aos serviços OTT em pequenos ISP atinge em média $52 \%$ do tráfego total do ISP.

Palavras-Chave: OTT, CDN, internet, provedor internet.

\section{INTRODUÇÃO}

A Internet representa uma força transformadora de paradigmas, fazendo com que situações cotidianas como a forma de assistir filmes e interagir com a sociedade tivessem uma rápida transformação. Como exemplo, os programas de televisão são estruturados em grades de programação definidos e apresentados em horários determinados, enquanto as plataformas de distribuição de vídeo on-line pela Internet, como o youtube e o Netflix, realizam a entrega do conteúdo mediante a escolha do usuário, de forma interativa e em variados dispositivos tecnológicos.

As plataformas de distribuição de vídeo on-line pela Internet consistem em sistemas distribuídos multiplataforma formando o denominado over the top - OTT. São serviços on-line que substituem a mídia tradicional e os serviços de telecomunicações. Consiste em um serviço multimídia que trafega por uma rede de terceiros, no qual o fornecedor do serviço OTT não tem responsabilidade sobre o meio de acesso ao serviço prestado. O termo OTT é utilizado para serviços que possuem servidores próprios para a distribuição via Internet, alguns exemplos são: a TV por IP; rádio sobre IP; mensagens instantâneas, ligações audiovisuais; vídeo on demand - VOD; entre outros. Neste contexto está à origem do termo OTT, no qual é possível um celular navegar em um site da mesma forma que um computador. Diversos dispositivos se 
conectam sem a necessidade de serem tecnologicamente similares. Exemplos de serviços OTT estão mostrados na figura 1.

Figura 1 - Tipos de serviços de OTT

\section{Movies \& TV NETFLIX Doyplay Youtube đ́iTunes amazon hulu

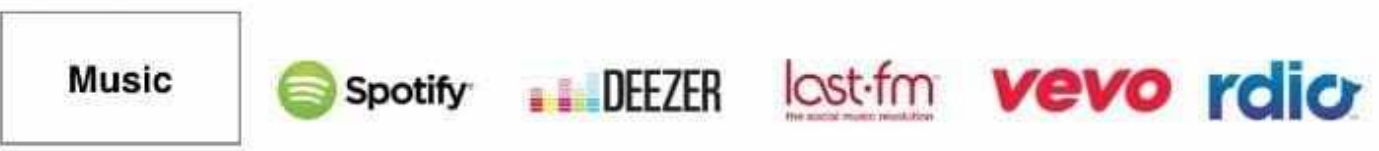

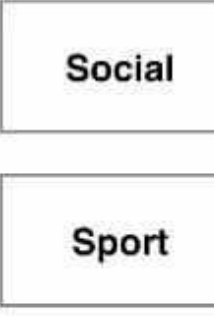

Productivity

Health \&

Fitness

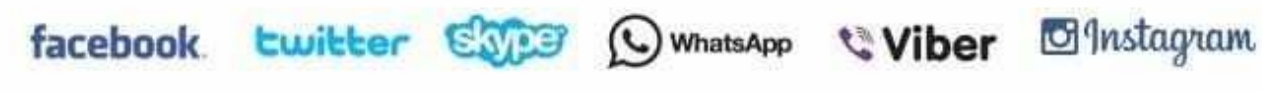

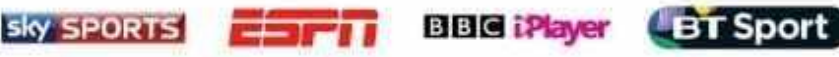
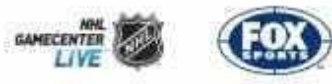

\section{Dropbox}

\section{jevernote}

III $\oplus$ XXxpensify

Google maps

Linked in

GARMIN̂

\section{mapmy 8 run}
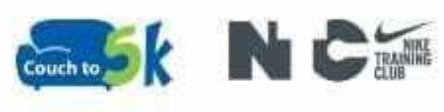

lumosity

Fonte: OTT SOURCE, 2013

O procedimento de distribuição, em uma plataforma OTT, é chamado de streaming e possui diversas formas de envio dependendo da necessidade de cada usuário. Para que ocorra o streaming é necessário seguir uma arquitetura. Nela existem camadas que influenciam cada momento do envio devido aos procedimentos que são seguidos. Para cada tipo de envio, processa-se um padrão, dependendo de critérios como o tipo de transmissão, a qualidade de serviço, os protocolos de envio, servidores que armazenam a mídia e, por fim, quais os dispositivos que irão receber e quais os aplicativos irão transmitir o conteúdo. 
Devido a falta de implantação de multicast na Internet, a distribuição de conteúdo OTT em larga escala é um grande desafio. Diversas formas vêm sendo utilizadas para tornar possível a distribuição de multimídia ao vivo em larga escala, como streaming adaptável, o uso de redes par-a-par (P2P) e as Rede de Distribuição de Conteúdo (Content Delivery Network - CDN).

O envio dos conteúdos de vídeo sobre a Internet necessita de diversos dispositivos de infraestrutura, associados aos provedores de serviços de Internet (Internet Service Providers - ISP), que tem que disponibilizar o enlace de acesso à Internet com largura de banda suficiente para garantir a qualidade do serviço. A alta disponibilidade do acesso e a velocidade de transmissão de dados, que pode variar de $256 \mathrm{Kbps}$ (Cabo ou Linha de assinante digital - DSL) até 1 Gbps em Fiber To The Home - FTTH, são características importantes da Internet de banda larga que influenciam o comportamento dos usuários.

O avanço da adoção das Tecnologias da Informação e Telecomunicações - TIC está diretamente relacionado ao desenvolvimento e expansão da infraestrutura e à oferta de acesso à Internet proporcionada pelos ISP. Em relação ao mercado atendido pelos provedores, a maioria dos ISP oferece seus serviços para o mercado privado (90\%) e domiciliar (88\%). Os pequenos provedores, com menos de 50 mil acessos, assumem papel fundamental, pois eles que atendem prioritariamente o mercado domiciliar $(91 \%$, frente a $75 \%$ das empresas ISP de médio porte e $51 \%$ das empresas ISP de grande porte). $\mathrm{Na}$ qualidade de intermediários que possibilitam o acesso à Internet, os provedores fornecem a plataforma para tecnologias de comunicação, desempenhando papel muito relevante para o desenvolvimento da Internet no país (CGI, 2016).

Assim, o crescimento do uso da Internet de banda larga, liderado pelo aumento de tráfego gerado pelos operadores de OTT, gera preocupações para os ISP, pois muitas das aplicações impõem uma carga de trabalho caracterizada por sessões de longa duração com um tráfego de dados intenso e contínuo (KURBALIJA, 2016). 
Segundo Sandvine, 2016, em seu relatório "Global Internet Phenomena", em 2016, O streaming de áudio e vídeo representava $71 \%$ do tráfego noturno nas redes de acesso fixo da América do Norte. A Sandvine espera que esse número alcance $80 \%$ até 2020. A adição de chamadas de vídeo e voz está gerando crescimento em aplicativos de comunicação em redes móveis na América Latina e América do Norte. Na América Latina, a quota de tráfego da WhatsApp é agora de $7,4 \%$, mais do que o triplo do que era há dois anos. Na América Latina, o Facebook e o Google representam mais de $70 \%$ do tráfego móvel total na região - de $60 \%$ no ano passado. Mais de $60 \%$ do tráfego móvel na América Latina e América do Norte está criptografado. A figura 2 mostra as 10 aplicações com maiores tráfegos na Internet na América Latina em 2016.

Figura 2 - Percentual de uso por aplicação do tráfego total de Internet

\begin{tabular}{|l|r|l|r|l|r|}
\hline \multicolumn{2}{|c|}{ Upstream } & \multicolumn{2}{c|}{ Downstream } & \multicolumn{2}{c|}{ Aggregate } \\
\hline BitTorrent & $30.03 \%$ & YouTube & $28.48 \%$ & YouTube & $25.91 \%$ \\
\hline YouTube & $9.30 \%$ & HTTP - OTHER & $11.66 \%$ & HTTP - OTHER & $11.12 \%$ \\
\hline HTTP - OTHER & $7.59 \%$ & SSL - OTHER & $9.76 \%$ & BitTorrent & $10.06 \%$ \\
\hline Facebook & $6.72 \%$ & Netflix & $8.31 \%$ & SSL - OTHER & $9.28 \%$ \\
\hline SSL - OTHER & $6.19 \%$ & BitTorrent & $6.96 \%$ & Netflix & $7.45 \%$ \\
\hline Ares & $5.27 \%$ & Facebook & $5.10 \%$ & Facebook & $5.32 \%$ \\
\hline Skype & $2.53 \%$ & MPEG - OTHER & $2.28 \%$ & MPEG - OTHER & $2.10 \%$ \\
\hline Netflix & $1.97 \%$ & RTMP & $1.79 \%$ & RTMP & $1.66 \%$ \\
\hline Dropbox & $1.16 \%$ & Google Market & $1.69 \%$ & Google Market & $1.52 \%$ \\
\hline MPEG - OTHER & $0.92 \%$ & Flash Video & $1.60 \%$ & Flash Video & $1.46 \%$ \\
\hline & $71.69 \%$ & & $77.63 \%$ & & $75.87 \%$ \\
\hline
\end{tabular}

sandvine

Fonte: Sandvine, 2016

No Brasil, o tráfego de vídeo na Internet crescerá 3 vezes entre 2016 e 2021, uma taxa de crescimento anual composta de $23 \%$. O tráfego de vídeo da Internet chegará a 4,0 Exabytes por mês em 2021, acima de 1,4 Exabytes por mês em 2016. O tráfego total de vídeo na Internet (comercial e domestico combinado) será de $84 \%$ de todo o tráfego da Internet em 2021, acima de 68\% em 2016. O tráfego de vídeo do consumidor domestico na Internet será de $86 \%$ do total de tráfego gerado pelo consumidor em 2021, ante 72\% em 2016 (CISCO, 2017). 
Nesse contexto, os ISP e as operadoras de telecomunicações avançam na oferta de serviços OTT como complemento à oferta de TV paga. Além de garantir uma boa experiência do usuário utilizando de soluções que aumentem a velocidade de entrega, como a CDN, que, basicamente, salva o conteúdo estático em local mais próximo do usuário e, em alguns casos, pode definir melhor caminho para os dados trafegarem na rede utilizando-se de conexões ao Ponto de Troca de Tráfego - PTT. O PTT é uma solução de rede com o objetivo de viabilizar a conexão direta entre as entidades que compõem a Internet, os Sistemas Autônomos (AS). É uma infraestrutura compartilhada que é instalada em uma região para receber conexões dos AS denominado de peering, com o objetivo de aperfeiçoar o desempenho da Internet ao manter a troca de tráfego mais localizada entre diferentes redes diminuindo o número de saltos entres os AS. Uma vez conectados no PTT, seus membros podem acordar em fazer o peering com troca de tráfego multilateral (ATM) com todos os demais membros de forma aberta ou o peering com acordos de tráfego bilateral (ATB) de natureza seletiva ou restritiva.

Portanto, os ISP precisam aperfeiçoar o uso dos seus recursos para cumprir os acordos de níveis de serviço (Service Level Agreement - SLA) estabelecidos com seus clientes e exigidos pela agencia reguladora ANATEL, principalmente, respeitando o marco civil da Internet no quesito de neutralidade da rede, que não permite que ISP deem prioridade de tráfego a determinados sites ou aplicações em detrimento de outros (NETO et al, 2007). Os ISP precisam constantemente conhecer e monitorar a demanda de seus usuários para definirem sua infraestrutura, como o dimensionamento dos enlaces de acesso ao backbone da Internet via porta IP (Internet Protocol - IP) e/ou a utilização de conexões PTT e/ou utilização de CDN no ambiente do ISP ou via PTT. Isso visa reduzir custos, recuperar investimentos e, principalmente, contribuir para a inserção da população brasileira na Sociedade da Informação ou na Sociedade em Rede, como definido por Castells (1999).

\subsection{JUSTIFICATIVA}

As plataformas OTT tem modificado e impulsionado o tráfego na Internet. Serviços como o Netflix tem mudado a cara da TV americana. Também, a empresa tem 
revolucionado o consumo de dados nos Estados Unidos - EUA. Segundo estudo da Sandvine (2016), o serviço ocupou 37\% do tráfego de dados de Internet no país em 2015. Logo atrás, outra plataforma de vídeo: o YouTube, com 18\%. O estudo mostra que $61 \%$ de todo o tráfego de dados dos EUA em 2015 é de serviços de vídeo. O dado é potencializado por usuários que abandonaram a TV paga e migraram para o streaming, em um fenômeno chamado "cord-cutter" (cortar o cabo, em tradução livre), que dá nome a um tipo de usuário que deixa de pagar pela TV por assinatura para assistir a vídeos apenas pela Internet (SANDVINE, 2016).

Segundo Cisco (2017) na edição Visual Networking Index (VNI), divulgada em junho de 2017, globalmente, o tráfego de vídeo IP será de $82 \%$ de todo o tráfego de Internet do consumidor em 2021, ante 73\% em 2016 e 75\% em 2017. O tráfego global de vídeo IP crescerá três vezes de 2016 a 2021. Os vídeos ao vivo na Internet serão responsáveis por $17 \%$ do tráfego de vídeo da Internet até 2022 . O vídeo ao vivo crescerá 15 vezes de 2017 a 2022. As redes de fornecimento de conteúdo (CDNs) transportarão 72\% do tráfego da Internet até 2022, em comparação com 56\% em 2017 (CISCO, 2018).

Outro organismo internacional que acompanha os impactos do OTT no mundo é a União Internacional de Telecomunicações (ITU-T). O impacto econômico dos serviços OTT é uma área de estudo abrangida pela Questão 9/3 do Grupo de Estudos ITU-T 3.

No Brasil, não há estudos definitivos que mensurem o impacto das plataformas OTT, principalmente nos pequenos provedores, embora se espere que a situação não seja diferente da observada nos EUA e America Latina. Segundo estimativas do Núcleo de Informação e Coordenação do Ponto BR (NIC.br), serviços como YouTube, Netflix, Facebook e Google geram até $60 \%$ do tráfego de usuários residenciais no País.

Assim, é importante e necessário conhecer e monitorar a demanda dos serviços OTT dos usuários para que os pequenos ISP possam definir a melhor forma de atender essas atuais demandas. Os ISP precisam evitar a sobrecarga de suas redes em horários de alta demanda de acesso e recuperar gastos e investimentos a partir da 
melhora no uso de seus recursos, principalmente na forma de disponibilização dos serviços de OTT. A caracterização do comportamento dos usuários para serviços OTT pode contribuir para um melhor entendimento da interação dos usuários desses serviços com os ISP, permitindo os pequenos ISP a gerenciar a capacidade de seus recursos, ofertar novos serviços com melhor qualidade e manter sua base de clientes, pois clientes que recebem uma melhor experiência dos serviços OTT são menos propensos a mudar o provedor de banda larga (OVUM, 2016).

Uma das motivações para o estudo dos impactos do OTT no setor de pequenos provedores é contribuir com informações relevantes para a disseminação do acesso à Internet com qualidade e para a elaboração de políticas que beneficiem a população brasileira como um todo na sua inserção na Sociedade da Informação, pois os pequenos ISP são os responsáveis diretos no atendimento do mercado domiciliar no Brasil, principalmente em regiões não assistidas pelas grandes operadoras.

\subsection{MATERIAIS E MÉTODOS}

Durante a realização do estudo de caso foram monitorados, coletados e analisados os tráfegos de dados de 8000 a 10.000 usuários de um provedor de Internet banda larga via fibra óptica da cidade de Montes Claros/MG, no período de novembro de 2017 a abril de 2019. Esses usuários correspondem à totalidade dos assinantes banda larga existente no momento inicial e final da pesquisa.

Os dados analisados foram coletados em intervalos de 60 minutos, por 24 horas, por no mínimo uma semana, de forma a permitir monitorar as sazonalidades no uso da Internet em um período de sete dias antes e após a implantação dos CDN da Netflix, Facebook e Google. A principio, essas amostras são estaticamente suficientes, visto que se pode inferir que a utilização de banda pelos clientes é, geralmente, constante no seu cotidiano.

Os CDN da Netflix, Facebook e Google foram implantados, respectivamente, em 20/11/2017, 13/08/2018 e 10/10/2018. As capacidades dos enlaces internos para 
conexões com os servidores do Netflix, Facebook e Google são respectivamente, 20 Gbps, 20 Gbps e 15 Gbps.

Para a realização das análises e criação dos gráficos de tráfego foi utilizado o sistema de monitoramento de rede Paessler Router Traffic Grapher (PRTG), que usa o protocolo SNMP, e aplicativos legados nos roteadores de borda.

Os dois roteadores de borda têm, respectivamente, capacidade de 4 Gbps e 1,5 Gbps, totalizando 5,5 Gbps nos dois enlaces de acesso a Internet. Não é implementado nenhum condicionamento ou contenção no tráfego gerado pelos assinantes no ISP.

\section{RESULTADOS}

\subsection{TRÁFEGO DOS ASSINANTES ANTES E APÓS A ATIVAÇÃO DO CDN NETFLIX}

O CDN da Netflix foi implantado no provedor no dia 20 de novembro de 2017. As velocidades dos planos de acesso à Internet oferecidos aos clientes eram de $20 \mathrm{Mbps}$, 50 Mbps e 100 Mbps, com maior predominância do plano de 20 Mbps.

Observa-se na figura 3 que após essa data houve redução média no tráfego de downstream próximo de $600 \mathrm{Mbps}$ e de upstream permaneceu inalterado. $\mathrm{O}$ tráfego de downstream corresponde ao tráfego de entrada assinalado na legenda da figura 3. E o tráfego de upstream ao de saída.

Como o tráfego médio de downstream antes da implantação do CDN Netflix era próximo de $3 \mathrm{Gbps}$, pode-se inferir que o tráfego médio do Netflix representava, aproximadamente, 20\% (vinte por cento) do tráfego total de downstream e de 17,1\% do tráfego total do provedor. 
Figura 3 - Tráfego total antes e após a implantação do CDN da Netflix

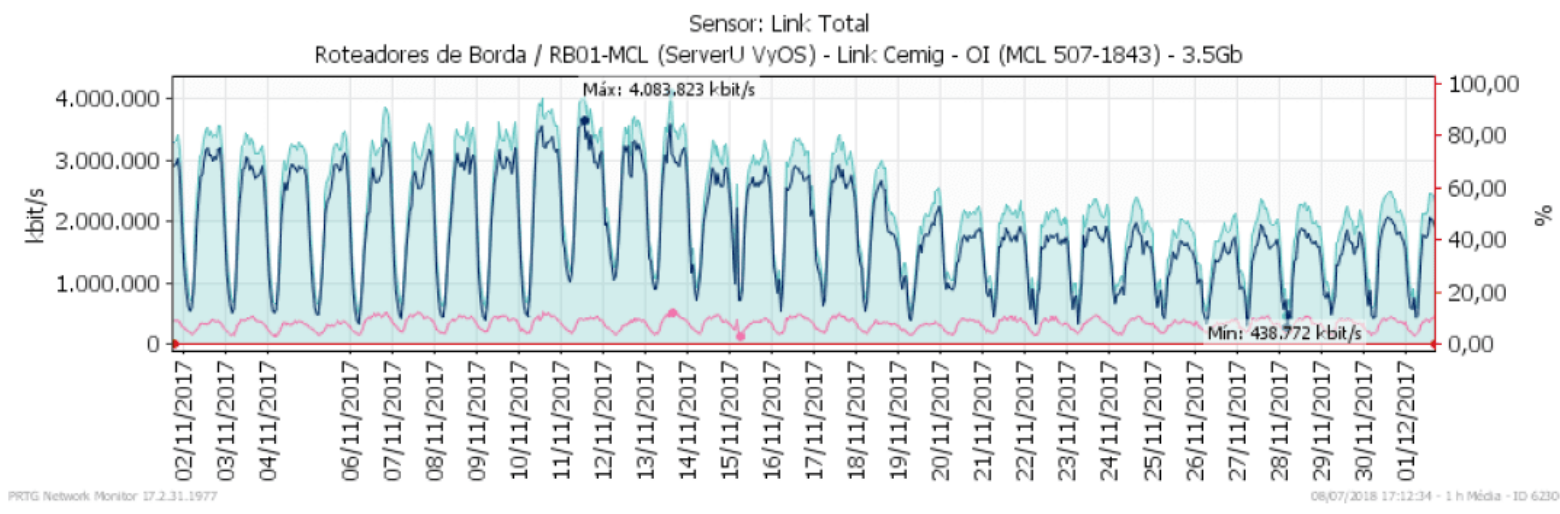

Tempo de inativid...(\%) \Tráfego total (kbit/s) $\square$ Tráfego de en... (kbit/s) \ Tráfego de saí... (kbit/s)

Fonte: Dados da pesquisa

Na figura 4 é apresentado o tráfego especifico do Netflix em outras datas. Na legenda o tráfego de entrada representa a utilização dos clientes, isto é, a velocidade de downstream dos clientes. E o tráfego de saída representa a velocidade utilizada pela Netflix para atualização dos seus servidores, que em média fica abaixo de $600 \mathrm{Mbps}$ e é realizado em períodos fora da maior utilização dos usuários de Internet do provedor.

Figura 4 - Tráfego especifico do Netflix em outros periodos

Netflix

Capacidade: 20Gbps / Download Máximo no período: 1,1 Gbps

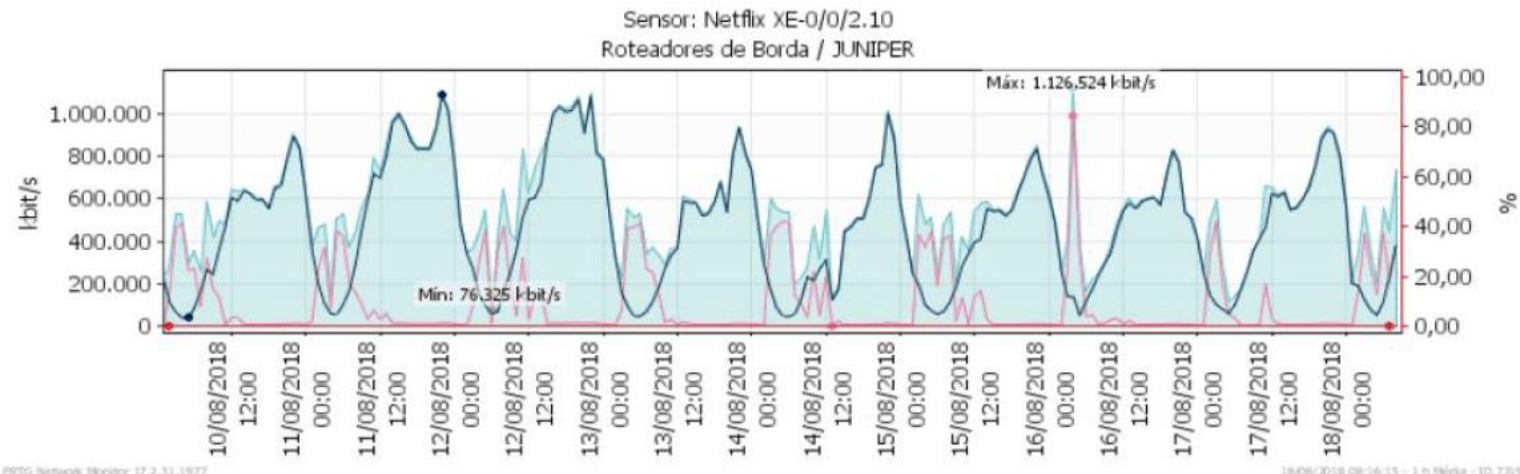

Tempo de inativid... (\%) — Tráfego total (kbit/s)

Tráfego de ent... (kbit/s) Tráfego de saida (kbit/s)

Fonte: Dados da pesquisa

RC: 36494

Disponível em: https://www.nucleodoconhecimento.com.br/ciencia-da-computacao/over-the-top 
Netflix

Capacidade: 20Gbps / Download Máximo no período: 1,16 Gbps

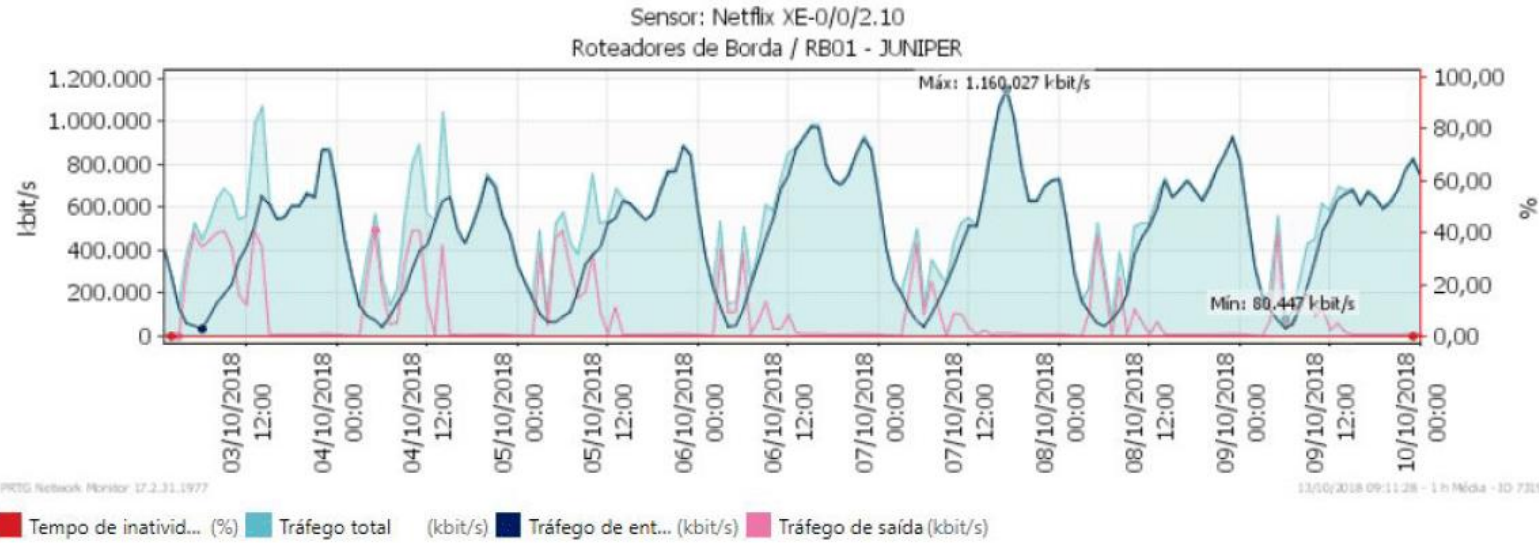

Fonte: Dados da pesquisa

\subsection{TRÁFEGO DOS ASSINANTES ANTES E APÓS A ATIVAÇÃO DO CDN FACEBOOK}

O CDN do Facebook foi implantado no provedor em 13 de agosto 2018, ou seja, após a ativação do CDN Netflix.

Observa-se na figura 5, que no período anterior a ativação, o dowload total máximo nos dois links foi de 3,95 Gbps. Neste período o total de clientes do provedor era próximo de 9.000 (nove mil) clientes. As velocidades dos planos de acesso a Internet oferecidos aos clientes eram de 50 Mbps, 100 Mbps e 120 Mbps, com maior predominância do plano de 50 Mbps.

Figura 5 - Tráfego total nos dois links antes e após a ativação do CDN Facebook

Operadora: Cemig

Capacidade: 1.5 Gbps / Download Máximo no período: 1,25 Gbps

Sensor: CEMIG-1.5G XE-0/0/0.1171

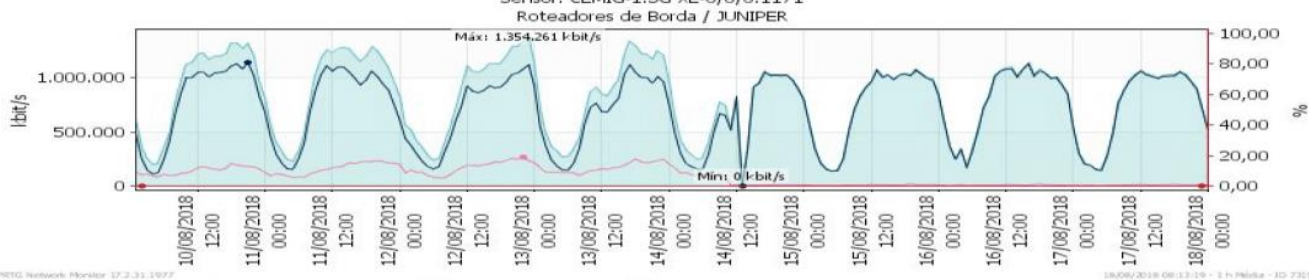

Disponível em: https://www.nucleodoconhecimento.com.br/ciencia-da-computacao/over-the-top 
Fonte: Dados da pesquisa

Operadora: OI

Capacidade: 4Gbps / Download Máximo no período: 2,7 Gbps

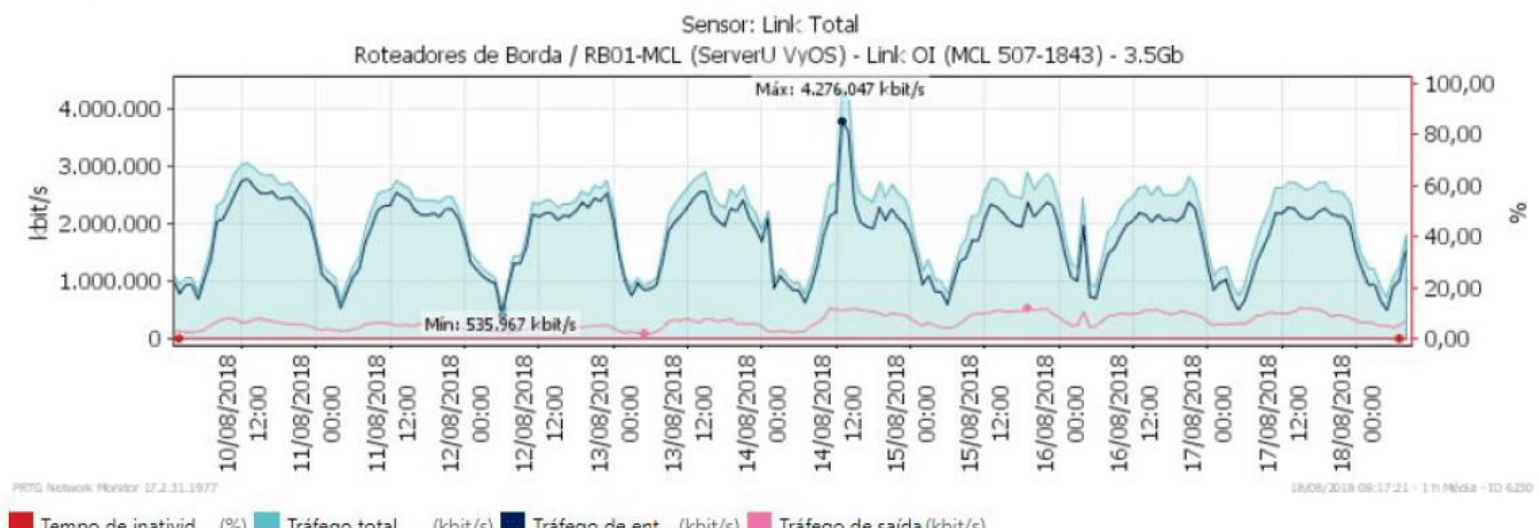

Fonte: Dados da pesquisa

Na figura 6 é apresentado o tráfego total do CDN Facebook. Observa-se que o tráfego médio deste CDN permaneceu próximo de $600 \mathrm{Mbps}$. Considerando o tráfego máximo de downstream dos dois links de 3,95 Gbps, pode-se inferir que o tráfego médio do CDN Facebook representava 15,2\% (quinze, dois por cento) do tráfego total de downstream do provedor. O tráfego de upstream do Facebook representa em médio $50 \%$ do tráfego de downstream.

Figura 6 - Tráfego do CDN Facebook

\section{Facebook}

Capacidade: 20Gbps / Download Máximo no período: $650 \mathrm{Mbps}$

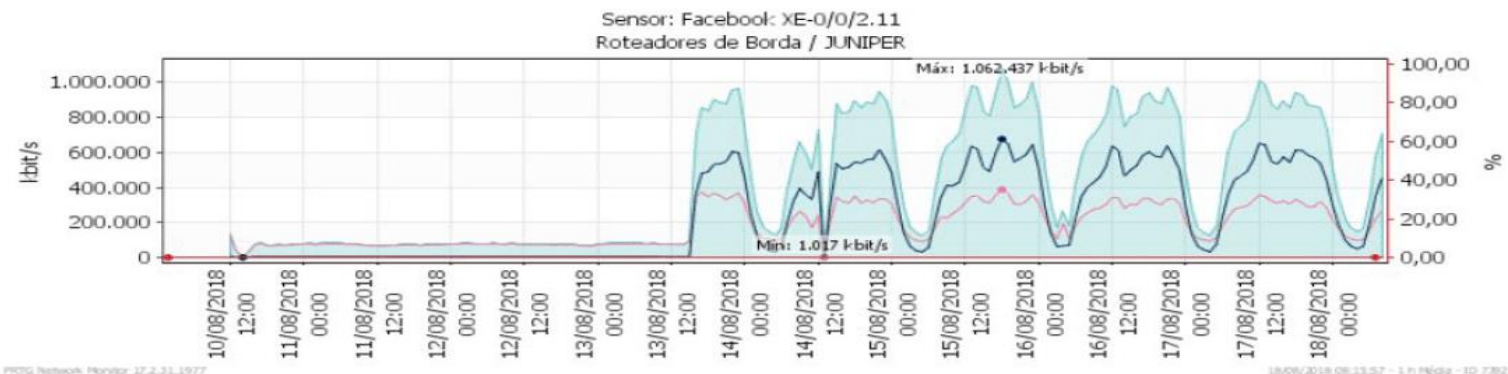

Tempo de inativid... (\%) Tráfego total (kbit/s) Tráfego de ent... (kbit/s) W Träfego de saida(kbit/s)

Fonte: Dados da pesquisa

RC: 36494

Disponível em: https://www.nucleodoconhecimento.com.br/ciencia-da-computacao/over-the-top 
Facebook

Capacidade: 20 Gbps / Download Máximo no período: 790 Mbps

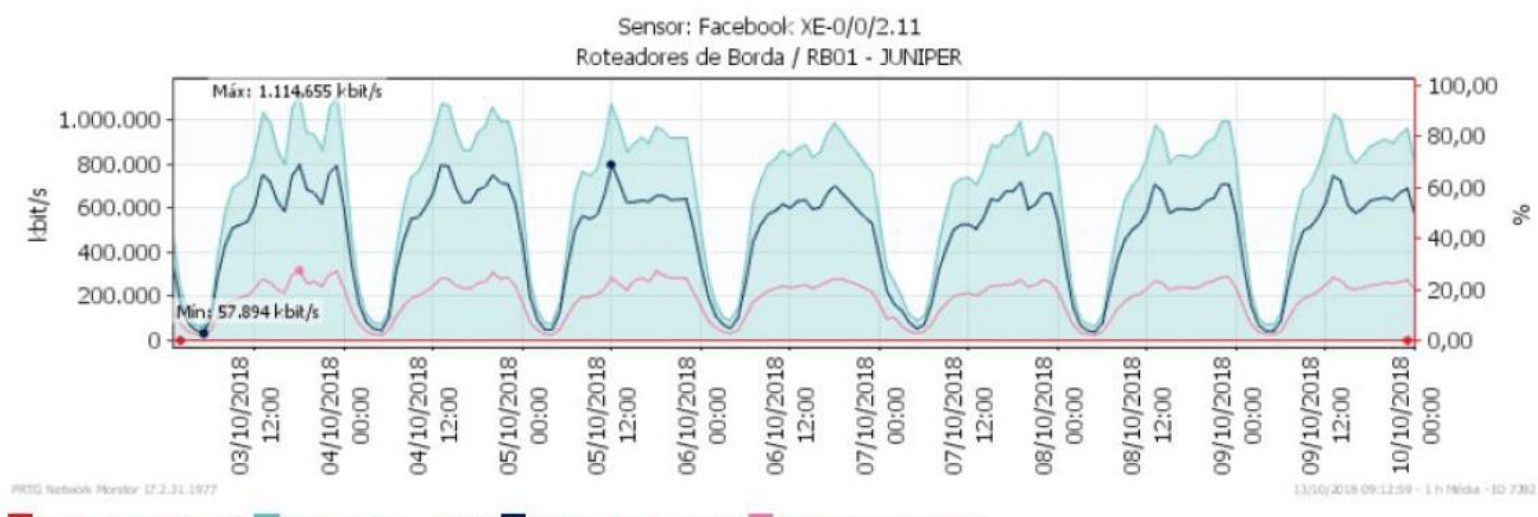

Tempo de inativid... (\%) — Tráfego total (kbit/s)

Tráfego de ent... (kbit/s) ㅁ Tráfego de saida (kbit/s)

Fonte: Dados da pesquisa

\subsection{TRÁFEGO DOS ASSINANTES ANTES E APÓS A ATIVAÇÃO DO CDN GOOGLE (INCLUSO YOUTUBE)}

O CDN do Google foi implantado no provedor em 11 de outubro de 2018, ou seja, após as implantações dos CDNs do Netflix e Facebook. Neste período o total de clientes do provedor era próximo de 9.200 (nove mil e duzentos) clientes. As velocidades dos planos de acesso a Internet oferecidos aos clientes eram de $50 \mathrm{Mbps}$, 100 Mbps e 120 Mbps, com maior predominância do plano de 50 Mbps.

Na figura 7 está representado o tráfego total dos dois links do provedor. Considerando o tráfego máximo de downstream nos dois links de 3,66 Gbps e o downstream máximo do Google de 660 Mbps (vide figura 8), pode-se inferir que o tráfego máximo do CDN Google representava $18 \%$ (dezoito por cento) do tráfego total de downstream do provedor. 
Figura 7 - Tráfego total nos dois links antes e após a ativação do CDN Google

Operadora: Cemig

Capacidade: 1.5 Gbps / Download Máximo no período: 861 Mbps

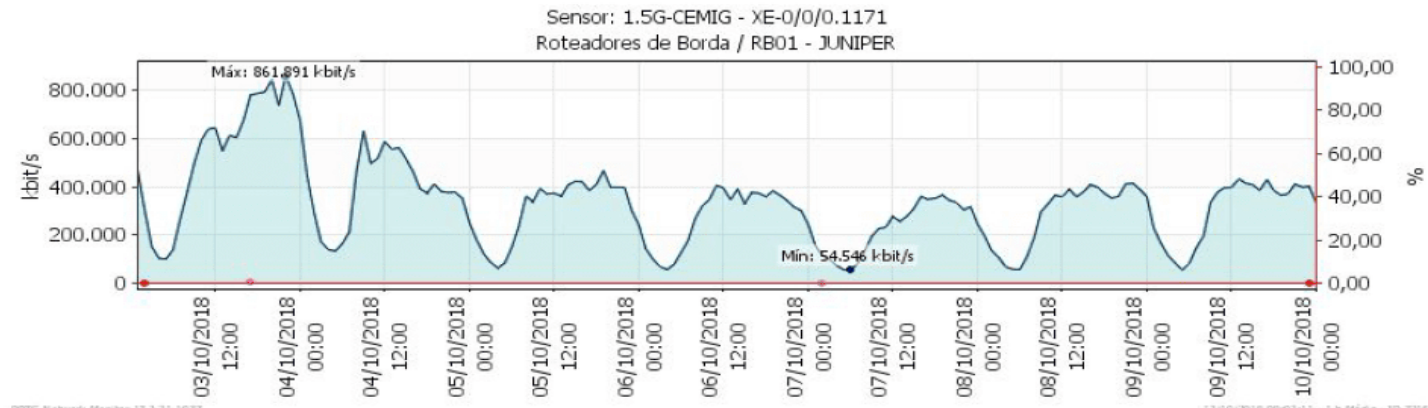

Tempo de inativid... (\%) — Tráfego total (kbit/s) Tráfego de ent... (kbit/s) $\square$ Tráfego de saída (kbit/s)

Fonte: Dados da pesquisa

\section{Operadora: $\mathrm{OI}$}

Capacidade: 4Gbps / Download Máximo no período: 2,8 Gbps

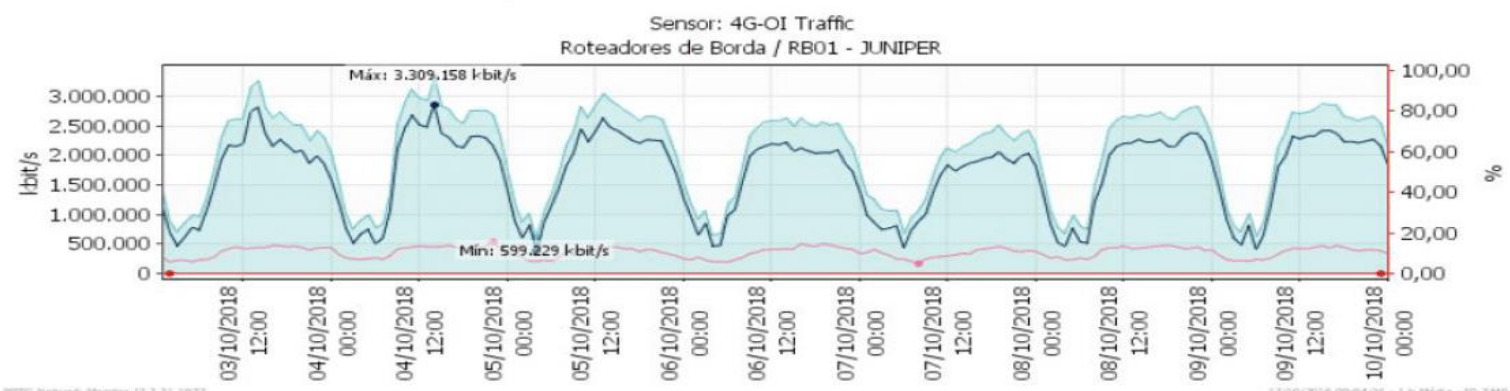

Tempo de inativid... (\%) Tráfego total (kbit/s) Tráfego de ent... (kbit/s) Trä́fego de saída (kbit/s)

\section{Figura 8 - Tráfego do CDN Google}

Google - (11/10 a 13/10)

Capacidade: 15 Gbps / Download Máximo no período: 660 Mbps

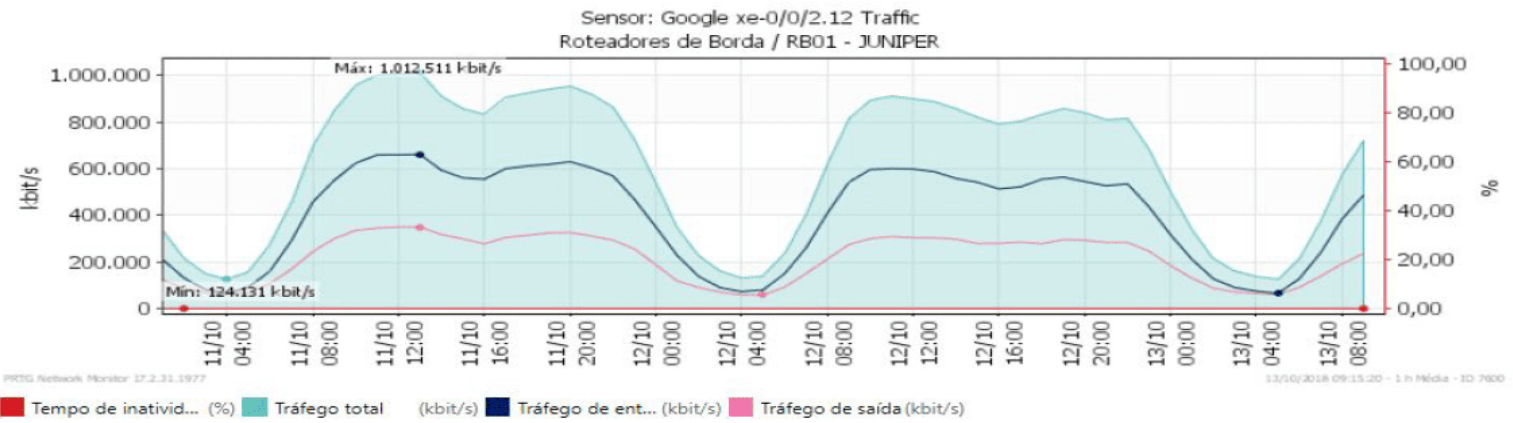

Fonte: Dados da pesquisa

RC: 36494

Disponível em: https://www.nucleodoconhecimento.com.br/ciencia-da-computacao/over-the-top 


\subsection{TRÁFEGO DOS ASSINANTES APÓS A ATIVAÇÃO DOS CDNS DO NETFLIX, FACEBOOK E GOOGLE}

Conforme dados da última pesquisa de (SANDVINE, 2018), em 2018, quase 58\% do tráfego de downstream da Internet é vídeo. Netflix continua no topo das aplicações de maior tráfego da Internet com 19,10\% (dezenove, dez porcento) de todo tráfego da Internet no continente Americano. No horário de pico em redes fixas, esse número pode chegar a $40 \%$ em algumas redes de operadoras na região. O Youtube representa $7,53 \%$ (sete, cinquenta e três porcento) e as redes sociais, incluindo Facebook, representa 5,1 (cinco, um porcento) de todo tráfego da Internet no continente. Também, segundo (CISCO, 2018) as CDNs transportarão 72\% do tráfego da Internet até 2022, em comparação com 56\% em 2017.

Com base nos dados das pesquisas da Sandvine e da Cisco e avaliado o tráfego do provedor, observa-se na figura 9 que não houve a necessidade de aumentar a capacidade final dos links. A figura 9 apresenta o tráfego total dos dois links do provedor 18 (dezoito) meses após a implantação do CDN Netflix. O provedor possuía mais 2.000 (dois mil) clientes em relação a data inicial da ativação dos CDNs, ou seja, representa o tráfego total de pouco mais de 10.000 (dez mil) clientes.

Mesmo com o aumento de mais de 2000 clientes e acréscimo de velocidades mínimas (mais de 5 vezes), nos planos oferecidos aos clientes pelo provedor, não houve necessidade de aumentar a capacidade final dos links. A velocidade máxima de downstream no periodo avaliado (mês de abril de 2019) não ultrapassou a velocidade máxima de downstream registrado quando da implantação inicial do CDN. Isso mostra a grande utilização dos CDNs no tráfego de downstream no provedor. No perido a velocidade de downstream máxima nos dois links foi de 2,8 Gbps. 
Figura 9 - Tráfego total nos dois links do provedor dezoito meses após a ativação dos CDNs

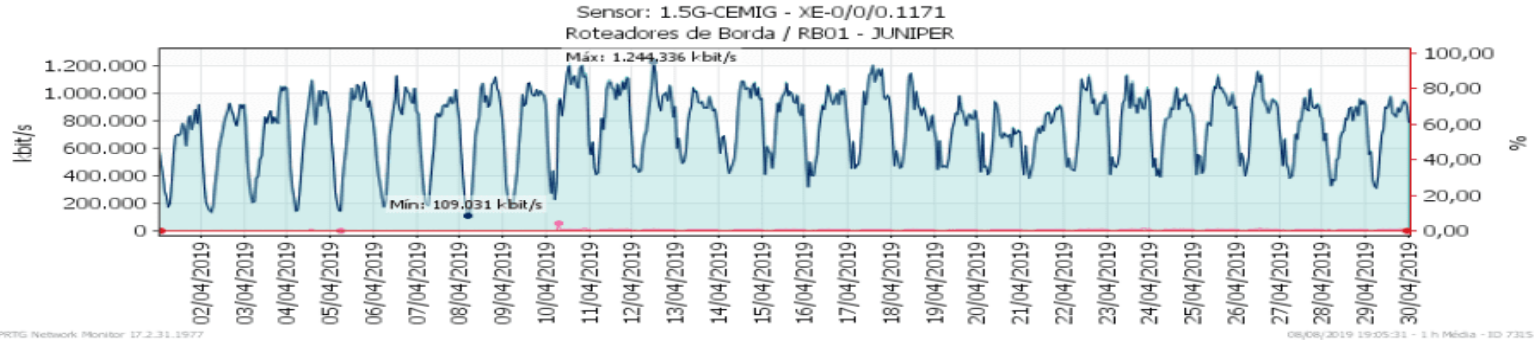

Tempo de inativid...(\%) — Tráfego total (kbit/s) —Tráfego de en... (kbit/s) — Tráfego de saí... (kbit/s)

Fonte: Dados da pesquisa

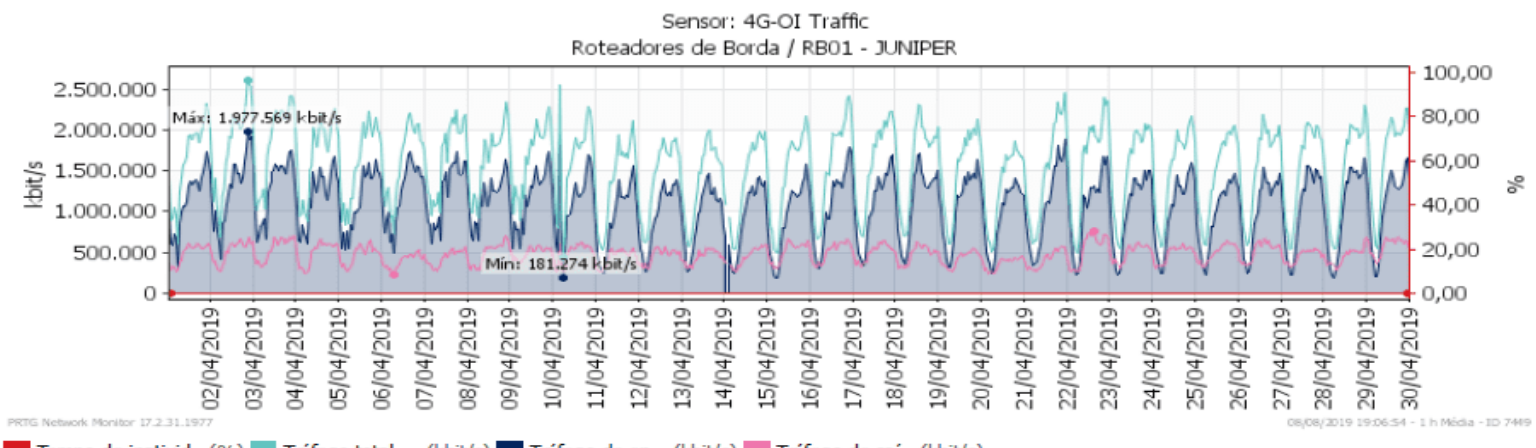

Fonte: Dados da pesquisa

Na figura 10, figura 11 e figura 12 estão representados, respectivamente, os tráfegos do Netflix, Facebook e Google no mês de abril de 2019.

Figura 10 - Tráfego do CDN Netflix

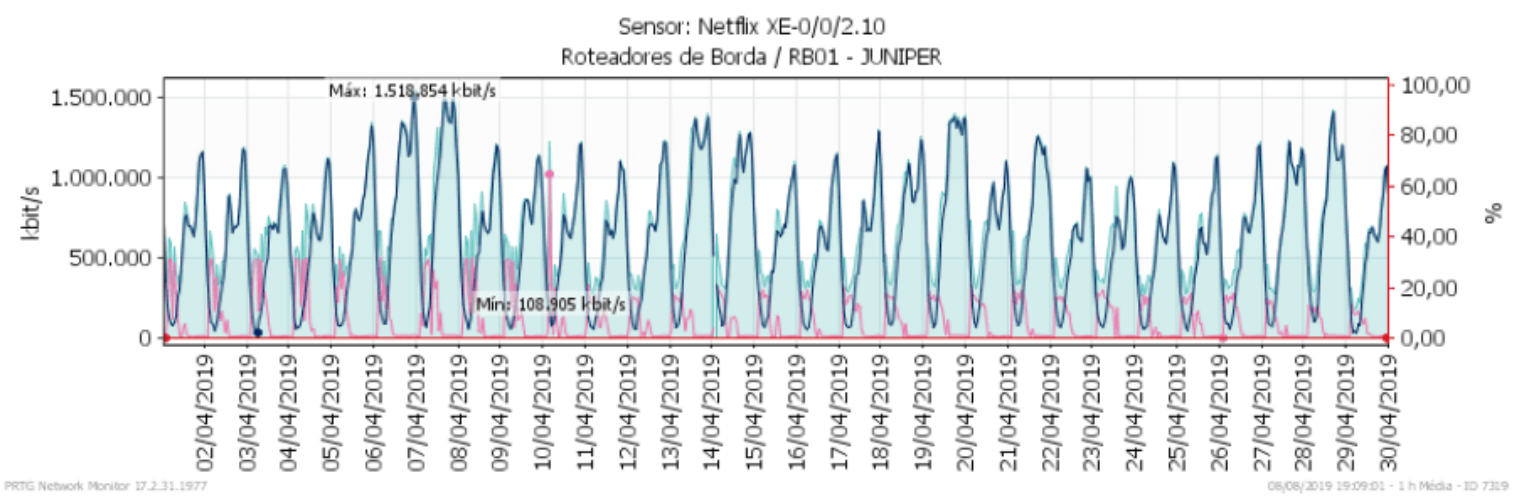

Tempo de inativid...(\%) — Tráfego total (kbit/s)

Tráfego de en... (kbit/s) \Tráfego de saí... (kbit/s)

Fonte: Dados da pesquisa

RC: 36494

Disponível em: https://www.nucleodoconhecimento.com.br/ciencia-da-computacao/over-the-top 
Figura 11 - Tráfego do CDN Facebook

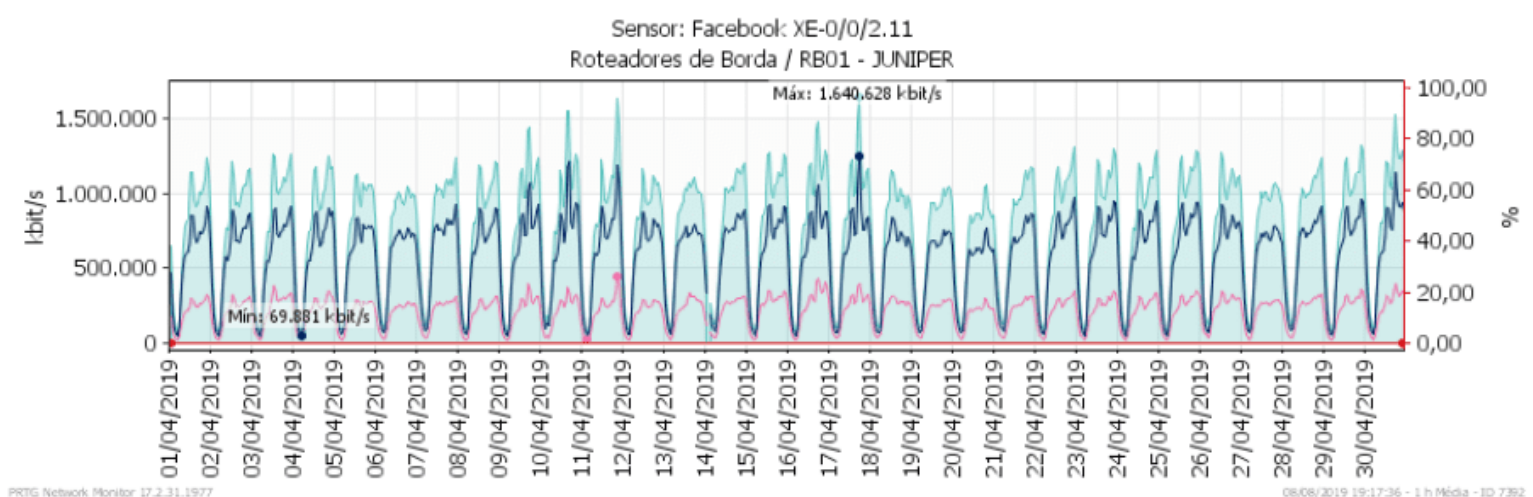

Tempo de inativid...(\%) Tráfego total (kbit/s) Tráfego de en... (kbit/s) Tráfego de saí... (kbit/s)

Fonte: Dados da pesquisa

Figura 12 - Tráfego do CDN Google

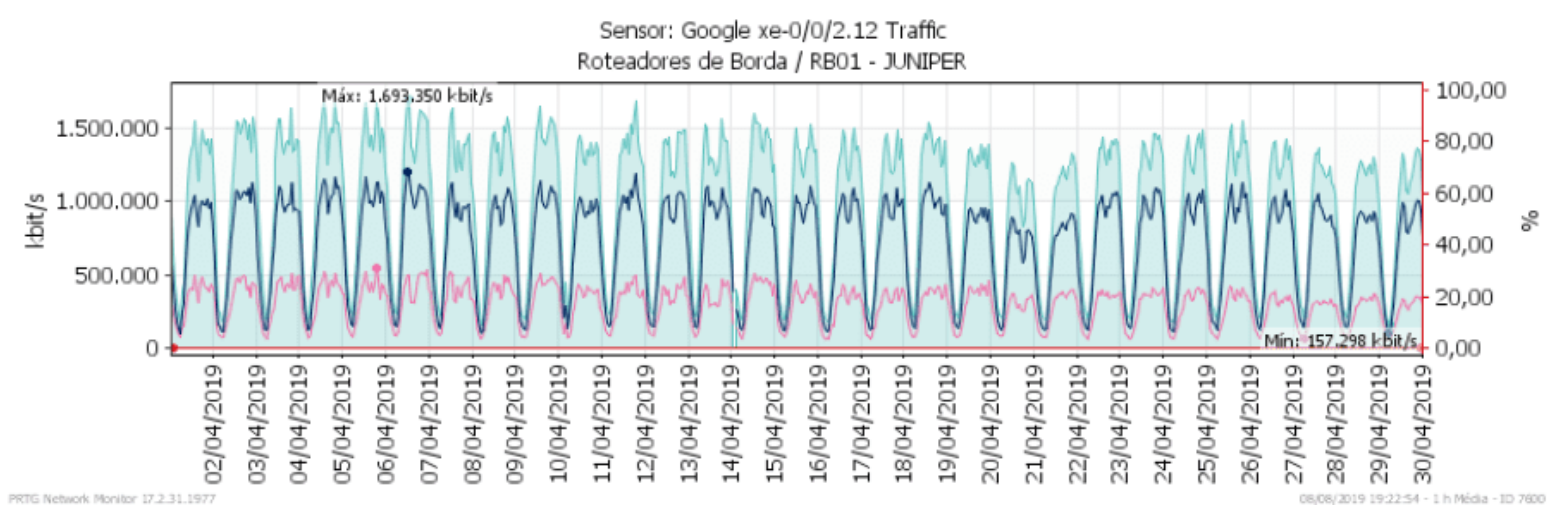

Tempo de inativid...(\%) Tráfego total (kbit/s) \Tráfego de en... (kbit/s) Tráfego de saí... (kbit/s)

Fonte: Dados da pesquisa

Os tráfegos médios no período do CDN Netflix foi 724,07 Mbps para o tráfego total e 623,34 Mbps para o tráfego downstream, com valor máximo atingindo a 1,16 Gbps no periodo noturno compreendido entre 20:00 hs e 23 hs.

Para o mesmo período, os tráfegos médios do CDN Facebook foi 788,97 Mbps para o tráfego total e 578,83 Mbps para o tráfego downstream, com valor máximo atingindo a 912 Mbps no periodo noturno compreendido entre 20:00 hs e 22:00 hs. 
Os tráfegos médios no período do CDN Google foi 1,0 Gbps para o tráfego total e 710,55 Mbps para o tráfego de downstream, com valor máximo atingindo a 1,2 Gbps nos periodos compreendidos entre 10:00 hs as $15: 00$ hs e 18:00 as 23:00 hs.

Os tráfegos médios dos dois links foi de 2,33 Gbps para o tráfego total e 1,84 Gbps para o tráfego de downstream, com valor máximo atingindo 2,80 Gbps. Esses valores representam todo tráfego gerado pelos clientes que não estejam direcionados aos três CDNs.

Assim, pode-se deduzir que o tráfego total médio gerado pelos clientes do provedor atinge 4,84 Gbps (2,33 Gbps + 724,07 Mbps + 788,97 + $1 \mathrm{Gbps})$ para o tráfego total e de 3,75 Gbps (1,84 Gbps + 623,34 Mbps + 578,83 Mbps + 710,55 Mbps) para o tráfego downstream.

Com base nesses dados, infere-se que os tráfegos dos CDNs Netflix, Facebook e Google representam próximo de $52 \%$ do tráfego total médio gerado pelos clientes, com participação individual de 15\% para o Netflix, 16\% para Facebook e 21\% para o Google (incluso youtube). Esses valores correspondem as estimativas do NIC.br, na qual estabelece que serviços como YouTube, Netflix, Facebook e Google geram até $60 \%$ do tráfego de usuários residenciais no País.

\section{CONSIDERAÇÕES FINAIS}

Não é surpresa que o streaming de vídeo seja o melhor tipo de aplicativo na Internet, assocido as redes sociais. O vídeo continua a impulsionar o uso da largura de banda de downstream.

Como os pequenos ISP são os responsáveis diretos no atendimento do mercado domiciliar no Brasil, principalmente em regiões não assistidas pelas grandes operadoras. Eles precisam entender a composição do tráfego (não o conteúdo) para que possam construir uma rede capaz de atender as expectativas de qualidade do consumidor, além de garantir uma boa experiência do usuário utilizando de soluções que aumentem a velocidade de entrega, como a CDN. 
Como resultado deste trabalho é possível ter uma referencia para o dimensionamento dos enlaces de acesso ao backbone da Internet via porta IP e a utilização de CDN no ambiente do ISP ou via PTT. O uso das CDNs local no ISP resulta em grande economia na contratação de links e ótima experiência de navegação na Internet pelos usuários.

O uso das CDNs nos pequenos provedores é altamente relevante para a disseminação do acesso à Internet com qualidade, permitindo o aumento da velocidade de acesso à Internet e a redução dos valores dos planos ofertados aos clientes, beneficiando a população brasileira como um todo na sua inserção na Sociedade da Informação.

\section{REFERÊNCIAS}

CASTELLS, Manoel. A sociedade em Rede. 8 ed. Rio de Janeiro: Paz e Terra, 1999. $639 p$, Volume 1.

CISCO. Cisco Visual Networking Index: Forecast and Methodology, 2016-2021. 2017. Disponível em: <http://www.cisco.com/c/en/us/solutions/collateral/serviceprovider/visual-networking-index-vni/complete-white-paper-c11-481360.pdf>. Acesso em 07/07/2017.

CISCO. Cisco Visual Networking Index: Forecast and Methodology, 2017-2022. 2018. Disponível em: < https://www.cisco.com/c/en/us/solutions/collateral/serviceprovider/visual-networking-index-vni/white-paper-c11-741490.html >. Acesso em 07/02/2019.

COMITÊ GESTOR DA INTERNET NO BRASIL - CGI. Pesquisa sobre o setor de provimento de serviços de Internet no Brasil [livro eletrônico]: TIC Provedores $2014=$ Survey about the Internet service provider sector in Brazil: ICT Providers 2014 / [coordenação executiva e ditorial/executive and editorial coordination, Alexandre $\mathrm{F}$. Barbosa; tradução para o inglês/ translation into English Prioridade Consultoria]. -- São Paulo: Comitê Gestor da Internet no Brasil, 2016. 987 Kb; PDF. Disponível em: 
<http://cetic.br/media/docs/publicacoes/2/TIC_Provedores_2014_livro_eletronico.pdf >. Acesso em 10/07/2017.

KURBALIJA, Jovan. Uma introdução à governança da internet [livro eletrônico] / Jovan Kurbalija; [Zoran Marcetic -Marca \& Vladimir Veljasevic; tradução Carolina Carvalho]. -- São Paulo: Comitê Gestor da Internet no Brasil, 2016. Disponível em < https://www.cgi.br/media/docs/publicacoes/1/CadernoCGlbr_Uma_Introducao_a_Go vernanca_da_Internet.pdf $>$. Acesso em 10/07/2017.

NETO, Marques, H. T., Almeida, V. A. F., and Almeida, J. M. Pricing broadband internet adaptive services. 15th International Symposium on Modeling, Analysis, and Simulation of Computer and Telecommunication Systems, 2007. MASCOTS'07. Pages 158-165.

OTT SOURCE 2013. OTT BLOG. Disponível em: < http://ottsource.com/ott-blog/>. Acesso em 05/07/2017.

OVUM. The Evolution of Big Video. Examining telco transformation video $\begin{array}{llll}\text { opportunities. } & 2016 . & \text { Disponível }\end{array}$ $<$ http://photos.prnasia.com/fileman/static_file/do_download?seq=1\&permalink=20160829/0861608784-a >. Acesso em 10/07/2017.

SANDVINE. The Global Internet Phenomena Reports, 2016. Disponível em: https://www.sandvine.com/trends/global-internet-phenomena/. Acesso em 05/07/2017.

SANDVINE. The Global Internet Phenomena Reports, 2018. Disponível em: https://www.sandvine.com/hubfs/downloads/phenomena/2018-phenomenareport.pdf. Acesso em 05/08/2019.

Enviado: Agosto, 2019.

Aprovado: Setembro, 2019. 